$10 / a^{25}-95850$

UCRL-ID-118509

\title{
Update on Diode-Pumped Solid-State Laser Experiments for Inertial Fusion Energy
}

\author{
C. Marshall \\ L. Smith \\ S. Payne
}

This is an informal report intended primarily for internal or limited extermal distribution. The opinions and conchunions atabed are those of the author and may or may not be thoce of the Laboretory.

Work performed under the auspices of the U.S. Department of Energy by the Lawrence Livermare National Laborabary under Contract W-7405-ENG-48.

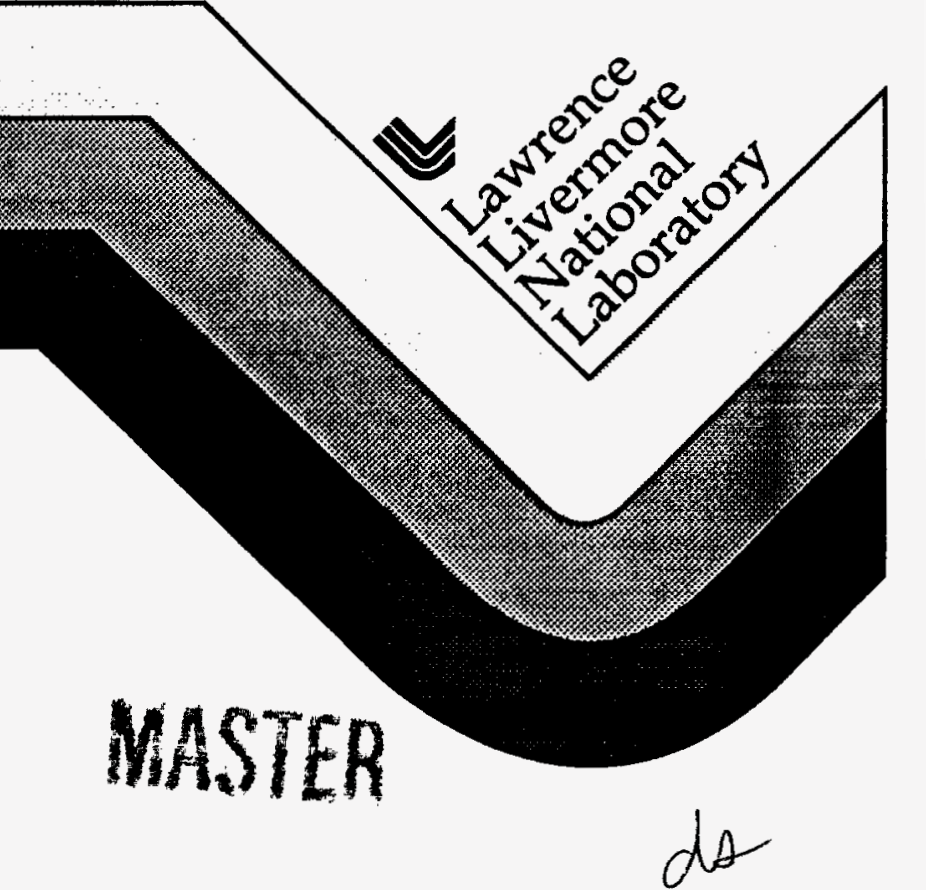




\section{DISCLAMMER}

This document was prepared 20 an socount of work sponsored by an agency of the United States Government. Neither the United States Government nor the University of California nor any of their employees, makes any warranty, express or implied, or assumes any legal liability or responsibility for the accuracy, completeness, or usefulness of any information, apparatus, product, or process disclosed, or represents that its use would not infringe privately owned rights. Reference herein to any specific commercial product, process, or service by trade name, trademark, manufacturer, or otherwise, does not necesearily constitute or imply its endorsement, recommendation, or favoring by the United States Government or the University of Californil. The views and opinions of authors expreaved herein do not neceasarily state or reflect those of the United States Government or the University of California, and shall not be used for advertising or product endorsement purposes.

This report hes been reproduced directly from the best avallable copy.

Available to DOE and DOE contractors from the Office of Scientific and Technical Information P.O. Box 62, Oak Ridge, TN 37831

Prices available from (615) 576-8401, FTS 626-8401

Avallable to the public from the

National Technical Information Service

U.S. Department of Commerce

5285 Port Royal Rd.

Springfield, VA 22161 
Interdepartmental letterhead

Mar'Station L- 493

August 8, 1994

Ext: $\quad 2-9781$

94-035

To: Howard Powell

CKe Ls 10

From: Chris Marshall, Larry Smith, and Steve Payne

Re: Update on diode-pumped solid-state laser experiments

for inertial fusion energy

\section{Executive Summary}

We have completed the initial phase of the diode-pumped solid-state laser (DPSSL) experimental program to validate the expected pumping dynamics and extraction cross-sections of $\mathrm{Yb}^{3+}$-doped $\mathrm{Sr}_{5}\left(\mathrm{PO}_{4}\right)_{3} \mathrm{~F}(\mathrm{Yb}: \mathrm{S}-\mathrm{FAP})$ crystals. Yb:S-FAP crystals up to $25 \times 25 \times 175 \mathrm{~mm}$ in size have been grown for this purpose which have acceptable loss characteristics $(<1 \% / \mathrm{cm})$ and laser damage thresholds $\left(-20 \mathrm{~J} / \mathrm{cm}^{2}\right)$. The saturation fluence for pumping has been measured to be $2.2 \mathrm{~J} / \mathrm{cm}^{2}$ using three different methods based on either the spatial, temporal, or energy transmission properties of a $\mathrm{Yb}: S-F A P$ rod. The small signal gain under saturated pumping conditions was measured. These measurements imply an emission cross section of $6.0 \times 10^{-20} \mathrm{~cm}^{2}$ that falls within error bars of the previously reported value of $7.3 \times 10^{-20} \mathrm{~cm}^{2}$, obtained from purely spectroscopic techniques. The effects of radiation trapping on the emission lifetime have been quantified and have been shown to lead to emission lifetimes as long as $1.7 \mathrm{~ms}$ for large optically dense crystals. The long lifetime of Yb:S-FAP has beneficial effects for diode-pumped amplifier designs, relative to materials with equivalent cross sections but shorter lifetimes, in that less peak pump intensity is required (thus lower diode costs) and that lower spontaneous emission rates lead to a reduction in amplified spontaneous emission. Consequently, up to $1.7 \mathrm{~J} / \mathrm{cm}^{3}$ of stored energy density was achieved in a 6x6x44 mm Yb:S-FAP amplifier rod; this stored energy density is large relative to typical flashlamp-pumped Nd:glass values of 0.3 to $0.5 \mathrm{~J} / \mathrm{cm}^{3}$. A $2.4 \mathrm{~kW}$ peak power InGaAs diode array has been fabricated by Beach, Emanuel, and co-workers which meets the central wavelength, bandwidth, and energy specifications for our immediate experiments. These results further increase our optimism of being able to produce a $\sim 10 \%$ efficient diode-pumped solid state laser for inertial fusion energy.

University of California U Lawrence Livermore
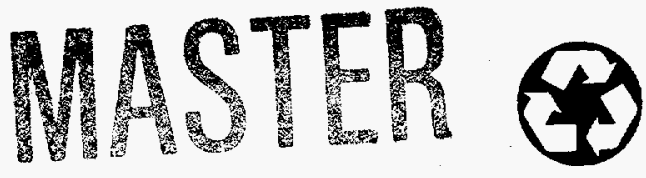


\section{Introduction}

Although flashlamp-pumped Nd:glass lasers have served as the main means by which the physics of inertial confinement fusion has been investigated over the last two decades, it had not been clear whether solid-state laser technology could meet the challenging requirements of a driver for an inertial fusion energy (IFE) power plant. Solid-state lasers have been criticized predominantly on the basis of offering inadequate cost and electrical to optical efficiency for IFE, although many other issues are regarded as being potentially problematic as well. For example, these include thermal management of the laser media, beam quality, a suitable high efficiency gain medium, and final optic survivability in the vicinity of a high yield target. In response to these and other concerns, we have endeavored to develop and validate a solid-state laser design that is consistent with IFE objectives and requirements.

Emmett, Krupke, et. al. first envisioned solid-state laser architectures having appropriate characteristics for IFE in the early 1980 's. ${ }^{1}$ Experimental work was first pursued several years later, when Albrecht, Sutton, and co-workers demonstrated a new strategy for cooling the solid-state amplifier slabs in fusion lasers. ${ }^{2}$ Their approach entails using a flow of helium gas across the optical aperture of the laser slab. The thermal and optical physics associated with this methodology was thoroughly investigated and quantitatively modeled on the basis of controlled thermo-optical experiments. Later, with IFE objectives in mind, a program was defined to identify a gain media well suited to IFE objectives, and the $\mathrm{Yb}^{3+}$-doped $\mathrm{Sr}_{5}\left(\mathrm{PO}_{4}\right)_{3} \mathrm{~F}$ (Yb:S-FAP) crystal emerged from this effort. ${ }^{3,4}$ The Yb:S-FAP material properties are found to be especially well-matched to the output of laser diode pump sources, and to other IFE requirements. Another crucial development involved a conceptual solution to the socalled final optic problem, where Chase and others examined the scientific literature and were able to deduce that heated fused silica should be able to sustain the anticipated neutron flux from the target without excessive discoloration. ${ }^{5}$ During the time while the gas-cooled slab technique, Yb:S-FAP gain medium, and heated fused silica final optic were being developed, a continuous and dramatic decrease in the cost of laser diodes occurred based on the work by R. Solarz and his group at LLNL and others elsewhere.

Most recently, Orth and co-workers have conceptually assembled these innovations, along with other detailed analyses and paradigms accepted by the ICF laser and plasma physics community, to describe the performance of a diode-pumped solidstate laser (DPSSL) within the IFE context. ${ }^{6,7}$ To perform this task, an extensive computer code was written and exercised. The results of these calculations have been 


\section{DISCLAIMER}

Portions of this document may be illegible in electronic image products. Images are produced from the best available original document. 


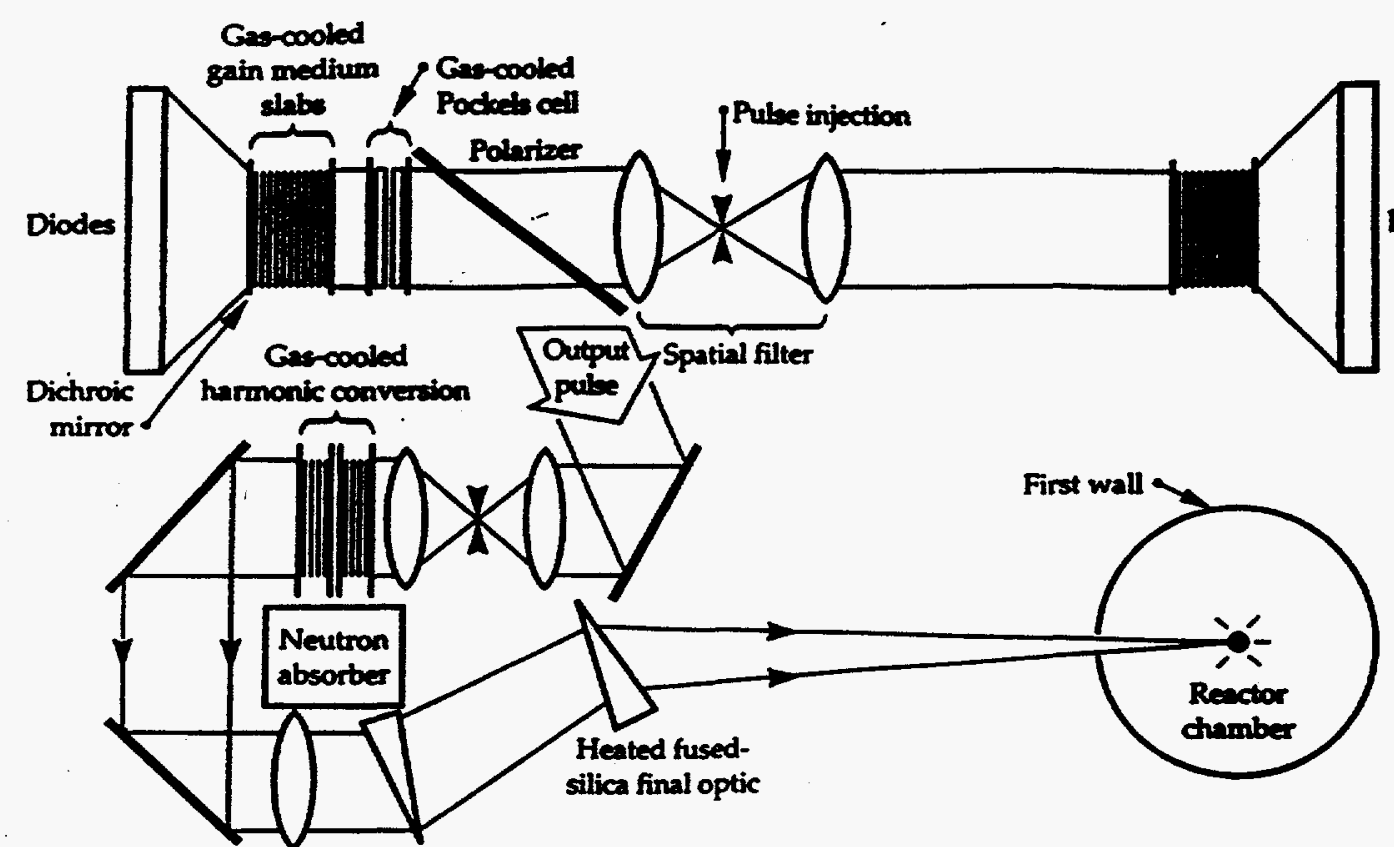

Figure 1. Component sketch of a single beamline from a conceptual diode-pumped solidstate laser based driver for an inertial fusion energy power plant recently developed by Orth and co-workers. ${ }^{6}$

encouraging and we have therefore been moving into the next step of development. In particular, we have begun to construct and study a small prototypical version of a $\mathrm{Yb}: \mathrm{S}-\mathrm{FAP}$ DPSSL, as discussed in detail in this memo. The Yb:S-FAP gain medium is particularly well adapted for this application in large part due to the high operational laser efficiency (expected to be $\sim 10 \%$ electrical to optical at $3 \omega$ ) that is possible, adequate thermal properties for $\sim 10 \mathrm{~Hz}$ repetition rate operation, and moderately large ( $-5-10 \times 10^{-}$ $20 \mathrm{~cm}^{2}$ ) cross-sections suitable for efficient pumping and extraction. Figure 1 is a sketch of one beamline for such a power plant adapted from reference ${ }^{6}$. The design is based upon Beamlet/NIF architecture with a four pass regenerative amplifier and a plasmaelectrode Pockels cell. The conceptual DPSSL driver design includes the use of a diodepumped $\mathrm{Yb}$ :S-FAP crystalline gain media that is expected to operate at a substantially higher efficiency than is possible with flashlamp-pumped Nd:glass.

One crucial goal of the IFE DPSSL project for this year is the experimental development and validation of efficient diode pumping and energy extraction in $\mathrm{Yb}: S-F A P$. The success of this project will enhance the credibility of laser driven ICF by demonstrating a laser-based driver technology that has the requisite efficiency to produce commercial power at a practical cost. The remainder of this memo will summarize recent experimental results obtained in the pursuit of developing efficient diode-pumped 
Yb:S-FAP amplifier capable of providing $\sim 10 \%$ wall plug efficiencies. In particular, we report on the pumping dynamics and small signal gain of Yb:S-FAP and the status of a prototype 900 -nm diode array suitable for pumping Yb:S-FAP.

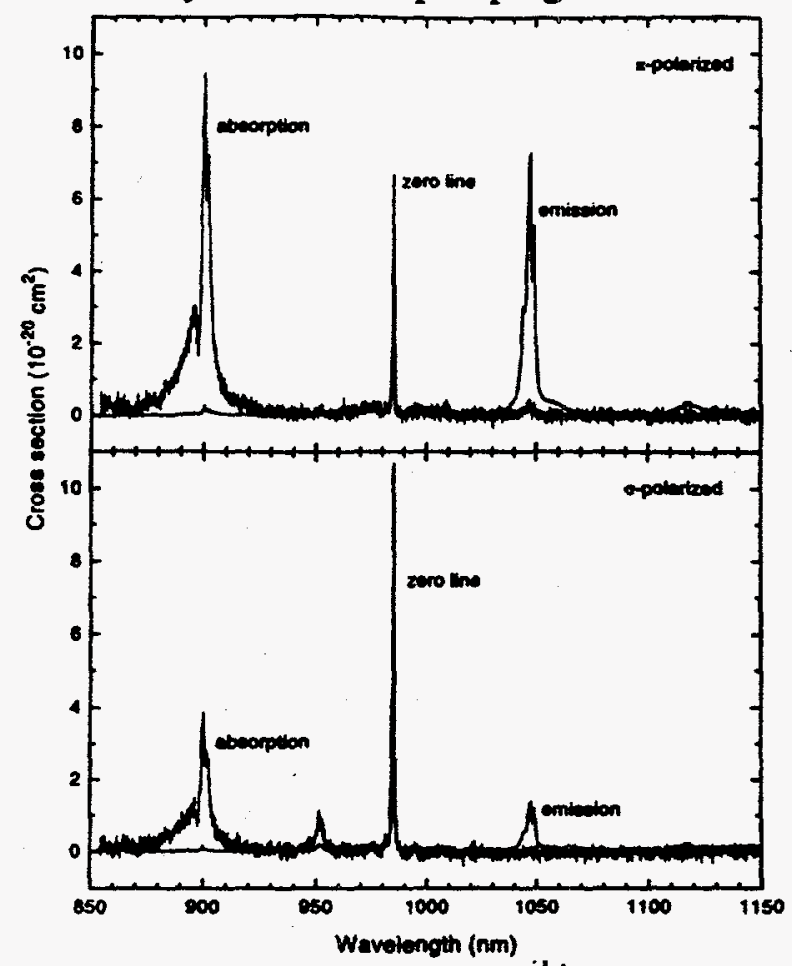

Figure 2. Absorption and emission spectra of $\mathrm{Yb}^{3+}$ doped $\mathrm{Sr}_{5}\left(\mathrm{PO}_{4}\right)_{3} \mathrm{~F}$ (S-FAP) for both $\pi$ and $\sigma$-polarizations along with a corresponding energy level diagram. ${ }^{3}$ Note that the primary absorption and emission peaks are predominantly $\pi$-polarized (parallel to the optical axis).

\section{Yb:S-FAP spectroscopic properties}

$\mathrm{Yb}: \mathrm{S}-\mathrm{FAP}$ lases at a wavelength $(1047 \mathrm{~nm})$ which is close to that of common $\mathrm{Nd}^{3+}$ doped materials such as LG-750 (1053 nm), YAG (1064 nm), and YLF (1047 and $1053 \mathrm{~nm}$ ). $\mathrm{Yb}: \mathrm{S}-\mathrm{FAP}$, however, is distinguished from $\mathrm{Nd}^{3+}$, however, by the simple electronic structure of $\mathrm{Yb}^{3+}$, in that it essentially has only two ground-state and excited electronic states, both of which are crystal field split into a straightforward quasi-three level arrangement. The absorption and emission spectra of $\mathrm{Yb}: \mathrm{S}-\mathrm{FAP}^{3}$ in Fig. 2 is suggestive of this simple structure. The inherently small quantum defect of $14 \%$, associated with the energy of the $900 \mathrm{~nm}$ pump and $1047 \mathrm{~nm}$ laser line, has aided in achieving the relatively large intrinsic laser slope efficiencies of $>70 \%{ }^{3,4}$ The emission lifetimes of $\mathrm{Yb}^{3+}{ }_{\text {-doped materials (of typically }}>1.0 \mathrm{~ms}$ ) also tend to be significantly longer than those for the same media doped with $\mathrm{Nd}^{3+}(<0.5 \mathrm{~ms})$, thereby offering 
practical advantages in lowered cost and increased effectiveness of diode laser pump sources by allowing for greater energy storage efficiency. One issue intrinsic to all $\mathrm{Yb}^{3+}$ based lasers is that they operate as quasi three-level systems because the terminal level is thermally populated at room temperature. For example, Yb:S-FAP has $~ 5 \%$ of its ground level population at room temperature in the terminal laser level at $1560 \mathrm{~cm}^{-1}$ above the ground state (see Fig. 2). If the pump source is sufficiently intense to effectively bleach the ground-state, one can see that the laser will operate as efficiently as a four-level system. Laser diodes are the obvious choice as a high irradiance pump source because the $900 \mathrm{~nm}$ pump region for Yb:S-FAP coincides with practical diode compositions. In addition, the relatively low saturation intensity for pumping of $2.0 \mathrm{~kW} / \mathrm{cm}^{2}$ is well suited to diode pumping because only moderate concentration of the laser diode array output is required to bleach the ground-state $\mathrm{Yb}$ population. This use of bleach-wave pumping ${ }^{8}$ is critical for efficient operation of a quasi three-level laser and is also desirable for high efficiency amplifier energy extraction. The above considerations taken as a whole suggest that diode-pumped solid-state $\mathrm{Yb}$-lasers may provide significant advantages over Nd-lasers for applications such as laser-driven IFE power plant designs, where high efficiency and high repetition rate are required.

\section{Crystal Growth of Yb:S-FAP}

In the past six months, high quality Yb:S-FAP boules up to 1" diameter by 7" long were grown for the first time by Prof. Bruce Chai at CREOL, under contract to LLNL using the Czochralski growth technique. Since the Yb:S-FAP crystals were cloudy immediately after growth, K. Schaffers and B. Chai have developed annealing techniques for Yb:S-FAP to entirely eliminate the scattering centers in the crystals. By post-growth annealing for several days at several hundred $\mathbf{K}$ below the melting point, it was possible to produce crystals with $<0.1 \% / \mathrm{cm}$ scattering loss and $<1 \% / \mathrm{cm}$ impurity absorption loss at the emission wavelength. These specifications are already suitable for efficient laser operation, although we will continue endeavoring to improve them. A forthcoming memo will be distributed that will describe in more detail the annealing process and physical processes involved. ${ }^{9}$

It is noteworthy that crystals produced by the above method were damage tested by F. Rainer and co-workers with very favorable results. Laser damage thresholds of $16.5 \pm 2.5 \mathrm{~J} / \mathrm{cm}^{2}$ (single shot) and $19.5 \pm 2.5 \mathrm{~J} / \mathrm{cm}^{2}$ (multiple shot) were observed with 1064-nm, 3-ns pulses. This shows that the multiple shot irradiation "conditions" or "laser anneals" the crystals and has been previously observed in KDP. These damage threshold are well above the DPSSL baseline design of $-10 \mathrm{~J} / \mathrm{cm}^{2}$ with $\sim 10-\mathrm{ns}$ pulses. 6,7 


\section{Cr:LiSAF pump laser}

While a diode array was being fabricated for our test amplifier, we used a freerunning Cr:LiSAF oscillator as a surrogate pump source to evaluate the pumping dynamics and efficiencies of Yb:S-FAP at a pump fluence level comparable to the initial DPSSL amplifier specifications. ${ }^{6,7}$ The use of the Cr:LiSAF pump laser provided the additional advantage of serving as a well-defined excitation source in terms of its bandwidth, beam profile, and temporal characteristics. The oscillator was run as an unstable resonator and employed a 6 by $80 \mathrm{~mm}$ Cr:LiSAF laser rod that was single flashlamp pumped. A dual-plate birefringent element was utilized for wavelength tuning. This laser, set to operate at $900 \mathrm{~nm}$ with a bandwidth of $<0.5 \mathrm{~nm}$, delivered up to $1.2 \mathrm{~J}$ of output energy for these experiments. Figure 3 displays the temporal output of the oscillator, with a 1/e pulse width of $250 \mu \mathrm{s}$. A $50-\mathrm{cm}$ lens was used to image relay the flat-top output of the oscillator into the Yb:S-FAP samples under test. The horizontal and vertical spatial profiles at the sample location (Figs. $4 a$ and $4 b$ ) had $1 / e$ beam diameters of 3.2 and $3.0 \mathrm{~mm}$, respectively. In addition, a variable wave-plate and polarizer were utilized to attenuate the beam for fluence-dependent experiments.

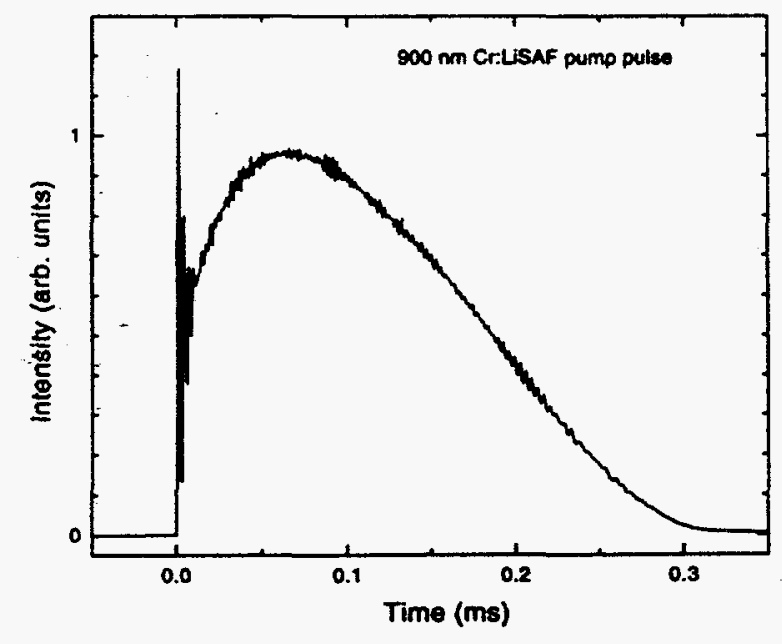

Figure 3. Temporal output pulse shape from the free running Cr:LiSAF oscillator that was used as a surrogate pump source for the laser diode array.

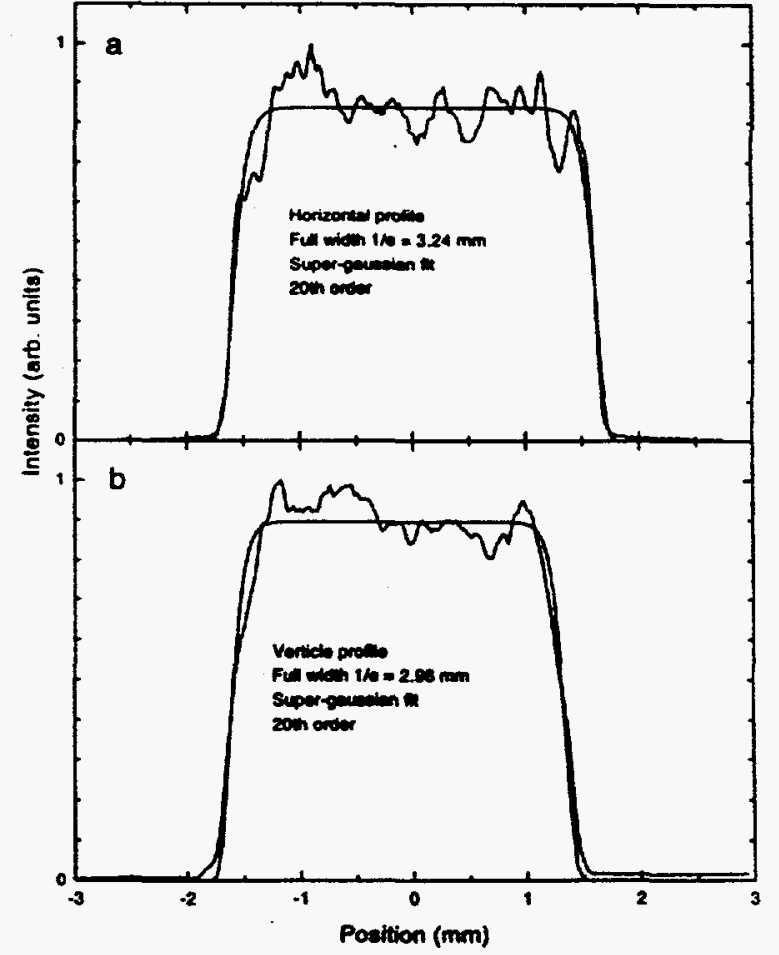

Figure 4. Spatial profile of $\mathrm{Cr}: \mathrm{LiSAF}$ oscillator $1.2 \mathrm{~J}$ pulse at the input of the sample test region. 


\section{Fluence-dependent pump transmission}

A $6 \times 6 \times 44 \mathrm{~mm}^{3}$ rectangular Yb:S-FAP crystal, with the c-axis (high-gain axis) set parallel to the $6 \times 6 \mathrm{~mm}$ square faces which had a $3^{\circ}$ wedge angle between the two square ends (to reduce parasitic oscillations), was utilized to investigate the pump dynamics, and the effect of the highly anisotropic gain and amplification. The two long sides with surface normal perpendicular to the c-axis were also ground to limit parasitic oscillation. A more detailed description of parasitic suppression, which is anisotropic due to the polarization-dependent emission shown in Fig. 2, is presented later.

The fractional transmission $T$ of the $\pi$-polarized pump light observed by pumping the wedged Yb:S-FAP parallelepiped along the long axis, with the Cr:LiSAF laser described above, is shown in Fig. 5. In the limit that the pump laser pulse length $(\sim 0.25 \mathrm{~ms})$ is much shorter than the lifetime of the Yb:S-FAP $(\sim 1.26 \mathrm{~ms})$ and that the absorption line is homogeneous, a straightforward Frantz-Nodvik analysis can be utilized to model the saturated pumping. In this limit the transmission $T$ is 10

$T=\frac{F_{\text {sat }}}{F} \ln \left[1+\left\{\exp \left(F / F_{\text {sat }}\right)-1\right\} \exp \left(-N_{o} h v L / F_{\text {sat }}\right)\right]$

where $\mathrm{F}$ is the fluence of the $900-\mathrm{nm}$ pump light (corrected for reflection losses), $F_{\text {sat }}$ is the pump saturation fluence, $N_{o}$ is the number density of $\mathrm{Yb}^{3+}$ ions, and $\mathrm{L}$ is the rod

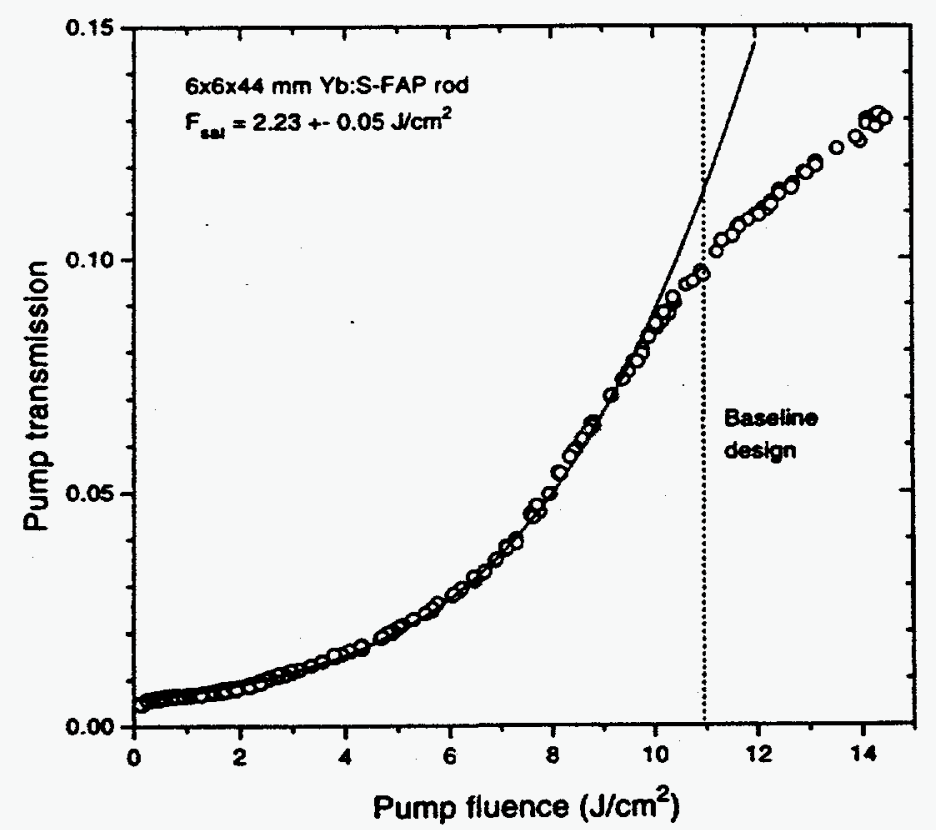

Figure 5. $900 \mathrm{~nm}$ pump transmission through a $44 \mathrm{~mm} \mathrm{Yb:S-FAP} \mathrm{rod} \mathrm{as} \mathrm{a} \mathrm{function} \mathrm{of}$ input fluence. The deviation of the modeling (solid line obtained using Eq. 1) from the experimental data above $11 \mathrm{~J} / \mathrm{cm}^{2}$ is due to parasitic oscillation losses. 
length. Using Eq. 1 it was possible to fit the transmission data in Fig. 5 up to $-10 \mathrm{~J} / \mathrm{cm}^{2}$ where the onset of soft parasitic modes produces a deviation from ideal behavior due to partial clamping of the excited-state population density which reduces bleaching. The presence of soft parasitic modes above $10 \mathrm{~J} / \mathrm{cm}^{2}$ is not unexpected since such parasitic modes are frequently encountered in other gain materials in rod configurations that have significant gain ( -10 single pass). The observed threshold for parasitics consistent with the maximum pump fluence that can be used for efficient operation of the rod amplifier geometry employed here. It is noteworthy that our baseline IFE design ${ }^{6}$ called for a maximum pump fluence of $\sim 11 \mathrm{~J} / \mathrm{cm}^{2}$. Consequently, we do not expect any significant problems from amplified parasitic losses within this operating regime for the experimental rod configurations we plan to study.

The pump saturation fluence was determined to be $2.2 \mathrm{~J} / \mathrm{cm}^{2}$ from a numerical fit shown in Fig. 5, which used Eq. 1 and two adjustable parameters, $N_{o}$ and $F_{\text {sat }}$. This experimental saturation fluence is in reasonable agreement with the predicted saturation fluence, defined as $h v / \sigma_{a b s}$, of $2.5 \mathrm{~J} / \mathrm{cm}^{2}$ derived from previously reported values for the absorption cross section. 2 The $\mathrm{Yb}^{3+}$ concentration of $N_{o}=1.3 \times 10^{19} \mathrm{~cm}^{-3}$ determined from the least squares fit is also in reasonable agreement with the expected concentration of $1.1 \times 10^{19} \mathrm{~cm}^{-3}$ determined from the absorption spectrum and the experimentally measured cross sections.

\section{Parasitic modes in the presence of anisotropic gain}

In the near future, we plan to totally internally reflect (TIR) the pump light in $\mathrm{Yb}$ :S-FAP laser rods with a divergent laser diode source (described later). For this purpose, it is desirable to have polished rod sides in a square geometry to allow for TIR with little depolarization (see polarized absorption in Fig. 2). However, it is also desirable to have ground surfaces on laser rods to inhibit the growth of parasitic modes. as is common in flashlamp pumped systems. Since S-FAP has anisotropic gain, it was thought that only some fraction of the sides may require ground surfaces to limit parasitic losses in the presence of relatively large single pass gains along the long axis of the rod. Consequently, a brief study was made of the onset of anisotropic soft parasitic oscillations as a function of rod surface configuration.

It was noted that the parasitic threshold, and consequently population clamping level, could be varied by up to a factor of three at a given pump fluence by selectively polishing or grinding different combinations of the four sides. When one or two of the faces with surface normals perpendicular to the c-axis were ground with $30 \mu \mathrm{m}$ grit rather than polished, the parasitic pump fluence threshold increased by up to two times and a 


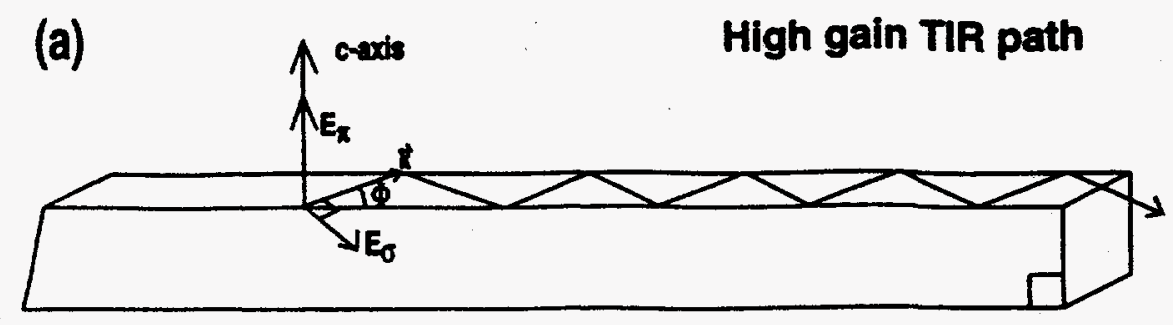

(b)

\section{Low gain TIR path}

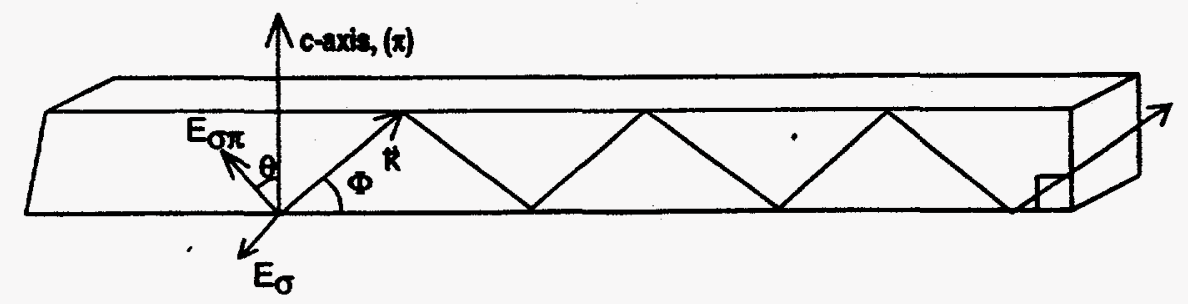

Figure 6. Yb:S-FAP rod geometry and polarized E-field orientations used to describe anisotropic parasitic amplification in the laser rod.

substantial increase ( $>2 \times$ compared to the predicted value) in the pump transmission was observed in the pump fluence range near $10 \mathrm{~J} / \mathrm{cm}^{2}$. However, when only one or two of the other sides, with surface normals parallel to the c-axis, were either ground or polished, no effects could be correlated with the preparation of the surfaces.

These observations can be understood by considering the highly polarized emission spectra shown in Fig. 2. Virtually all of the 1047-nm emission $(-70 \%)$ is concentrated in the $\pi$-polarized state (parallel to the c-axis of the crystal) which fosters a situation where $\pi$-polarized parasitic modes will tend to build up with wave-vectors that propagate primarily in the plane perpendicular to the c-axis (see Fig. 6a). Since the $\sigma$ polarized emission cross section at $1047 \mathrm{~nm}$ is much less than the $\pi$-polarized cross section (see spectra in Fig. 2), a ray with a wave-vector propagating at an angle in the plane defined by the $c$-axis and the long axis of the rod (see Fig. 6b) will experience relatively little amplified spontaneous emission (ASE) at the totally internally reflecting (TIR) critical angle. It turns out that this gain is actually smaller then the $\pi$-polarized gain propagating parallel to the long axis of the rod. A more quantitative treatment of the parasitic amplification discussed above follows.

The projection of the E-field vector onto the complex-index ellipsoid for a uniaxial crystal such as S-FAP can be written as 11

$\frac{1}{\left(n^{\prime}+i n^{\prime \prime}\right)^{2}}=\frac{\cos ^{2} \theta}{\left(n^{\prime}{ }_{\pi}+i n^{\prime \prime}\right)^{2}}+\frac{\sin ^{2} \theta}{\left(n_{\sigma}^{\prime}+i n^{\prime \prime}\right)^{2}}$, 
where $\theta$ is the angle the E-field vector makes with the optical axis, and $\tilde{n}=n^{\prime}+i n^{\prime \prime}$ is the complex index of refraction that is composed of two complex indices $\tilde{n}_{\pi}$ and $\tilde{n}_{\sigma}$ for the unique optical $(\pi)$ and two orthogonal $(\sigma)$ principal axes, respectively, (also referred to as the extraordinary and ordinary directions). The imaginary portion of the index of refraction carries the quantitative information on the magnitude of the polarized small signal gain, $G_{i}$, through the relation

$$
G_{i}=\exp \left(\alpha_{i} \ell_{\text {eff }}\right)=\exp \left(\left\{N_{\text {exc }} \sigma_{i}^{e m}-N_{g r} \sigma_{i}^{a b s}\right\} \ell_{\text {eff }}\right)=\exp \left(\frac{4 \pi n_{i}^{\prime \prime}}{n_{i}^{\prime} \lambda_{o}} \ell_{\text {eff }}\right),
$$

where $\ell_{e f f}$ is the effective path length in the medium, $N_{\text {exc }}$ is the excited-state number density, $\lambda_{o}$ is the wavelength of the emission line in vacuum, and $\sigma_{i}^{e m}$ is the emission cross section with polarizations $i=\pi$ or $\sigma$. In the limit that $n_{i}{ }^{\prime \prime} / n_{i}{ }^{\prime} \ll<1$, which is valid for typical $\mathrm{Yb}^{3+}$ number densities of $10^{19}$ ions $/ \mathrm{cm}^{3}$ and cross sections of $10^{-19} \mathrm{~cm}^{2}$ (i.e. $\left.n_{i}{ }^{\prime \prime} / n_{i}{ }^{\prime} \approx 10^{-4}\right)$, Eq. 2 can be reduced to $\sigma^{e m}(\theta)=\sigma_{\pi} \cos ^{2} \theta+\sigma_{\sigma} \sin ^{2} \theta$,

where $\sigma^{e m}(\theta)$ is the effective emission cross section.

Consider three propagation pathways, where each has either $\sigma$ or $\pi$ polarizations. Two directions are defined by the TIR cone with wave-vectors in the planes defined by the two adjacent faces of the rod as shown in parts (a) and (b) of Fig. 6, and the third wave-vector is considered to propagate straight down the long axis of the rod. With these six waves, it is possible to define the principal components of several limiting cases for parasitic gain or ASE. Table I provides a calculation of the small signal gain for these six limiting cases with $n_{\pi^{\prime}}=n_{\sigma^{\prime}}=1.62, \lambda_{o}=1047 \mathrm{~nm}, N_{g r}=N_{e x c}=0.6 \times 10^{19} \mathrm{~cm}^{-3}, \sigma_{\pi}^{e m}$ $=7.3 \times 10^{-20} \mathrm{~cm}^{2}, \sigma_{\sigma}{ }^{e m}=1.4 \times 10^{-20} \mathrm{~cm}^{2}, \sigma_{\pi} a b s=0.4 \times 10^{-20} \mathrm{~cm}^{2}, \sigma_{\sigma} a b s=0.0 \times 10^{-20}$ $\mathrm{cm}^{2}$ and longitudinal crystal length of $4.4 \mathrm{~cm}$, which were all chosen to estimate the conditions of the experiment shown in Fig. 5 . For a $\pi$-polarized ray propagating down the longitudinal axis of the rod, the small signal gain $\mathrm{G}$ is calculated to be 6.2 ; however, if the wave-vector of the amplified ray is tilted upward as in Fig. 6a, G falls to 4.4 even with $100 \%$ TIR and the corresponding $~ 60 \%$ increase in path length. Therefore, the top and bottom surfaces of the rods, as depicted in Fig. 6, do not require a ground surface to suppress parasitics. On the other hand, if an amplified ray TIRs off the front and back surfaces as in Fig. 6a, G increases to 19, indicating that a ground surface or an ASE absorber will aid in suppressing parasitic ASE. The other three cases outlined in the last three rows of Table I, are strictly $\sigma$-polarized and consequently have very little amplification. Cork-screw parasitic modes are also common in isotropic laser rods which 
are simply superpositions of the cases described above. For the anisotropic gain case discussed here, however, the gain coefficients of the cork-screw modes will fall in between the range of the limiting cases described above, and the polarized nature of the gain in Yb:S-FAP will disfavor these types of modes. In summary, the highly anisotropic nature of gain in Yb:S-FAP enables one to treat ASE as essentially a two-dimensional problem where $\pi$-polarized rays propagating in the plane perpendicular to the $c$-axis play the predominant role.

Table I. Estimates of the parasitic gain coefficients calculated using Eqs. 3 and 4 for three wave-vectors and two polarizations as pictured in Fig. 6 using a $\mathrm{Yb}^{3+}$ ground state number density of $1.2 \times 10^{19} \mathrm{gm}^{-3}$ with $50 \%$ inversion and polarized emission cross sections from the literature 3,4

\begin{tabular}{|l|l|l|l|l|l|}
\hline $\begin{array}{l}\text { Propagation } \\
\text { direction, and } \\
\text { polarization }\end{array}$ & $\begin{array}{l}\text { Angle } \\
\text { between } \\
\text { E-field and } \\
\text { c-axis, } \\
\theta \text { (degrees) }\end{array}$ & $\begin{array}{l}\text { Angle } \\
\text { between } \\
\text { wave-vector } \\
\text { and long axis } \\
\text { of crystal, } \Phi, \\
\text { (degrees) }\end{array}$ & $\begin{array}{l}\text { Effective ray } \\
\text { length, leff } \\
(\mathrm{cm}), \\
\{4.4 / \mathrm{cos} \Phi\}\end{array}$ & $\begin{array}{l}\text { Emission } \\
\text { absorption } \\
\text { cross section } \\
\text { at } 1047 \mathrm{~nm}, \\
\text { from Eq. } \\
\left(10^{-20} \mathrm{~cm}^{2}\right)\end{array}$ & $\begin{array}{l}\text { Calculated } \\
\text { small signal } \\
\text { gain from } \\
\text { Eq. } 4\end{array}$ \\
\hline $\begin{array}{l}\text { Straight through, } \\
\pi \text {-polarized }\end{array}$ & 0 & 0 & 4.4 & $7.3,0.4$ & 6.2 \\
\hline $\begin{array}{l}\text { TIR plane parallel } \\
\text { to c-axis (Fig 6b), } \\
\text { mixed } \pi \sigma-p o l .\end{array}$ & 52 & 52 & 7.1 & $3.6,0.1$ & 4.4 \\
\hline $\begin{array}{l}\text { TIR plane } \\
\text { perpendicular to } \\
\text { c-axis ( Fig 6a), } \\
\pi \text {-polarized }\end{array}$ & 0 & 52 & 7.1 & $7.3,0.4$ & 19 \\
\hline $\begin{array}{l}\text { Straight through, } \\
\sigma \text {-polarized }\end{array}$ & 90 & 0 & 4.4 & $1.4,0.0$ & 1.4 \\
\hline $\begin{array}{l}\text { TIR plane parallel } \\
\text { to c-axis (Fig 6b), } \\
\sigma \text {-polarized }\end{array}$ & 90 & 52 & 7.1 & $1.4,0.0$ & 1.8 \\
\hline $\begin{array}{l}\text { TIR plane } \\
\text { perpendicular to } \\
\text { c-axis ( Fig 6a), } \\
\sigma \text {-polarized }\end{array}$ & 90 & 52 & 7.1 & $1.4,0.0$ & 1.8 \\
\hline
\end{tabular}

\section{Spatial bleach-wave pumping}

We also studied the effects of Yb:S-FAP bleach-wave pumping by observing the spatially resolved emission strength along the long face of the $6 \times 6 \times 44 \mathrm{~mm} \mathrm{Yb:S-FAP} \mathrm{rod} \mathrm{with} \mathrm{a}$ $1.05 \mu \mathrm{m}$ bandpass filter and a CCD camera after excitation with the Cr:LiSAF laser described above. The camera was placed approximately 10 inches above the crystal and perpendicular to the beam propagation direction. The crystal was also masked to minimize stray light in the images. 


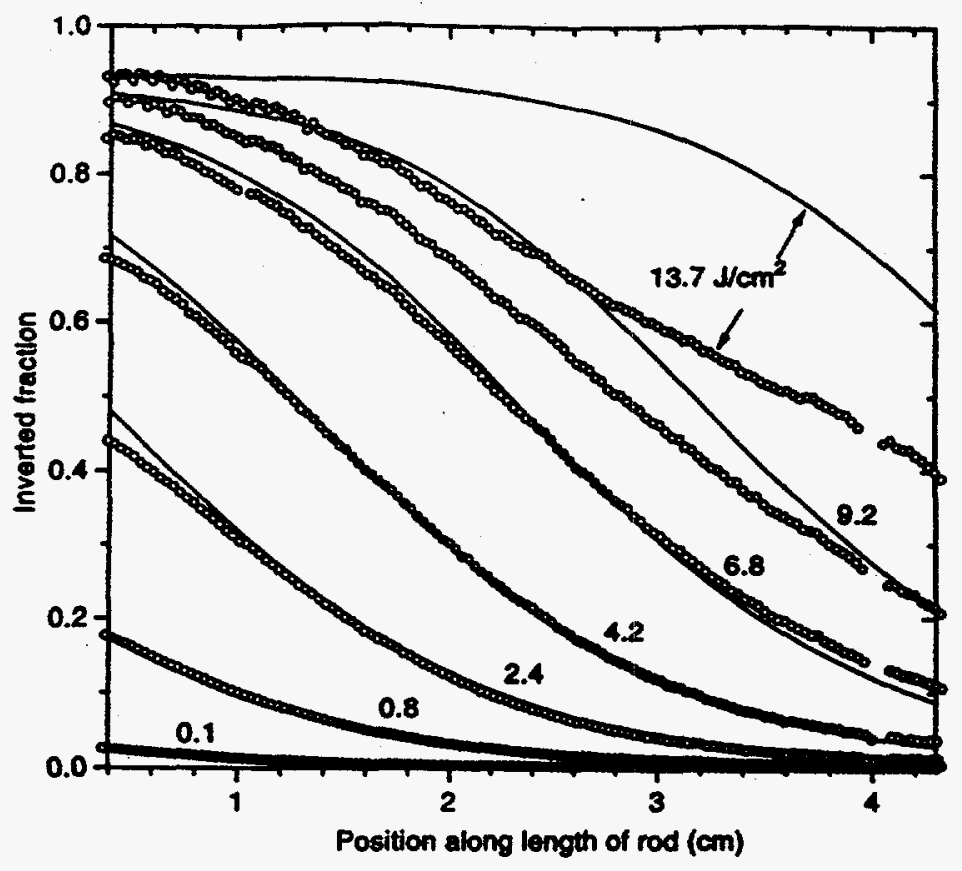

Figure 7. Spatial determination of excited states produced in a Yb:S-FAP rod plotted on a fractional population inversion scale for seven different 900 -nm pump fluences. Experimental points are drawn as open circles while the solid lines are numerical simulations. The deviation between data and modeling at the highest input fluences is due to parasitic oscillation in the rod.

The resulting data shown in Fig. 7 are longitudinal line-outs of the emission image averaged across $2 \mathrm{~mm}$ of the flat top portion of the pump beam. The end $4 \mathrm{~mm}$ of the images were truncated due to imaging artifacts from emission reflections off the $6 \times 6$ $\mathrm{mm}$ square ends of the rod. The solid lines in Fig. 7 are numerical simulations described below. The modeling includes the spatial and temporal evolution of the pump pulse as well as the temporal evolution of the $\mathrm{Yb}^{3+}$ energy levels at each longitudinal position along the rod. Both the upper and lower levels are assumed to thermalize instantaneously relative to the $0.2 \mathrm{~ms}$ timescale of the pumping experiment, effectively forming a two level system with no stimulated emission at the pump wavelength. Under these approximations, the equations governing ground and excited-state population densities become

$\frac{d N_{g r}}{d t}=\frac{-d N_{e x c}}{d t}=\frac{-\sigma_{a b s} N_{g r}(z, t) I(z, t)}{h v}+\frac{N_{e x c}(z, t)}{\tau_{e m}}$,

respectively, where $I(z, t)$ is the intensity of the pump field as a function of the longitudinal position $z$ and time $t, \tau_{e m}$ is the effective emission lifetime, and $h v$ is the 
energy per pump photon. The pump intensity attenuation is governed by the spatial and temporal dependence of the ground-state density via the relation

$\frac{d I(z, t)}{d z}=-N_{g r}(z, t) \sigma_{a b s} I(z, t)$.

To fit the spatial profiles in Fig. 7 , the pump pulse initial condition is assumed to be a plane wave with a $0.2 \mathrm{~ms}$ full width $1 / \mathrm{e}$ Gaussian temporal envelope. By utilizing different incident pump fluences, it was possible to reasonably match the bleach-wave data sets up to $\sim 9 \mathrm{~J} / \mathrm{cm}^{2}$ as can be seen in Fig. 7. The only adjustable parameter used in these fits arose from the need to convert the observed emission intensity data (which have arbitrary scaling) to population inversion fractions. The relative magnitudes of the emission intensity was preserved in that only one constant scaling factor was utilized for all the data curves shown in Fig. 7. No other adjustable parameters were utilized in these simulations, since the calculations used the same $900 \mathrm{~nm}$ absorption cross section $\left(9.5 \times 10^{-20} \mathrm{~cm}^{2}\right)$ and $\mathrm{Yb}^{3+}$ concentrations $\left(1.26 \times 10^{19} \mathrm{~cm}^{-3}\right)$ determined in the previous section from the saturation fluence measurements in Fig. 5 . For the case of $13.7 \mathrm{~J} / \mathrm{cm}^{2}$ incident fluence, however, there exists a significant deviation between the calculations and the inversion data across the entire length of the rod. This deviation again indicates the presence of soft parasitic oscillation above $\sim 10 \mathrm{~J} / \mathrm{cm}^{2}$ since no accommodation was made for these parasitic modes in the modeling. As seen before in the data of Fig. 5 parasitics are not observed to play an important role below $10 \mathrm{~J} / \mathrm{cm}^{2}$.

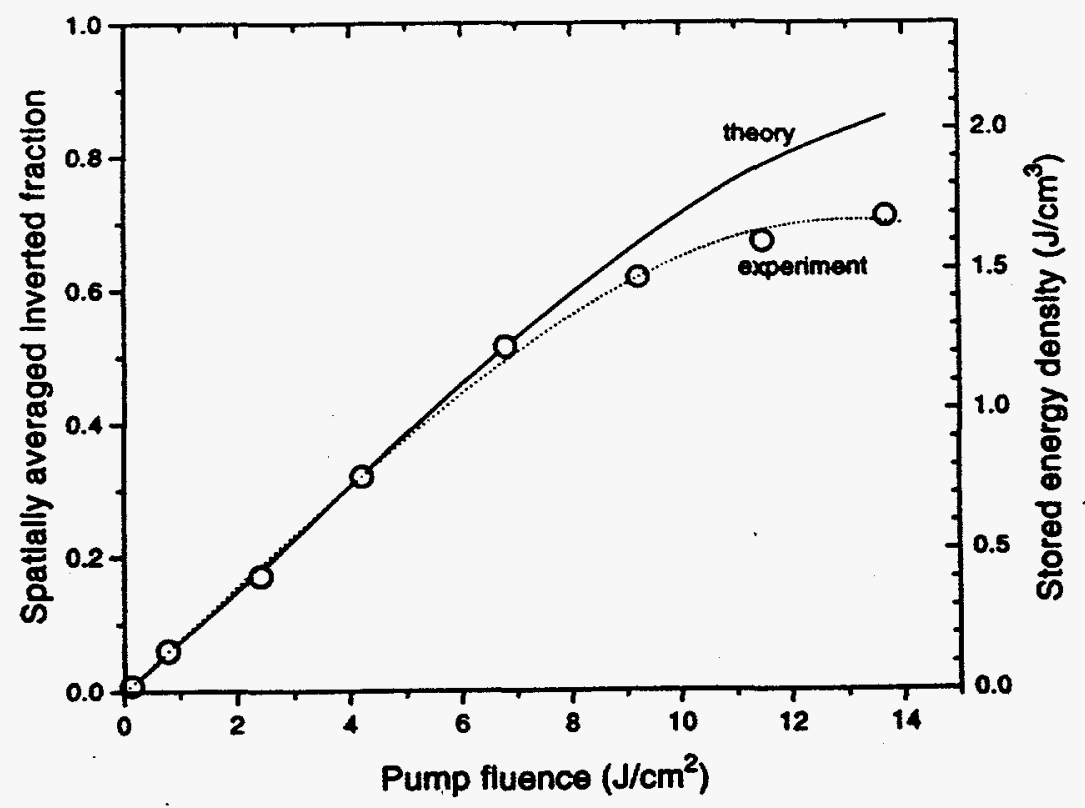

Figure 8. Spatially averaged inversion fraction, derived from the data and fits in Fig. 7, verses 900 -nm pump fluence. The stored energy density is shown on the right hand scale. 
By spatially integrating the data and modeling curves in Fig. 7, it is possible to determine the experimental and predicted (in the absence of parasitics) average inversion fraction in the rod. Figure 8 displays the spatially averaged inversion fraction obtained using this method. It is noteworthy that a spatially averaged maximum inversion fraction of $70 \%$ was experimentally produced. This leads to an average excited-state stored energy density of $1.7 \mathrm{~J} / \mathrm{cm}^{3}$ at our highest pump fluences. This is large compared with values commonly produced for typical flashlamp-pumped Nd:glass slab amplifiers $\left(0.3 \mathrm{~J} / \mathrm{cm}^{3}\right)$ and rod amplifiers $\left(0.5 \mathrm{~J} / \mathrm{cm}^{3}\right){ }^{12}$

\section{Temporal pump pulse saturation}

The temporal distortion of the pump pulse transmitted through the $44 \mathrm{~mm}$ long Yb:S-FAP rod was investigated by observing the Cr:LiSAF transmitted pump pulses with a photodiode through a 950-nm shortpass filter. The photodiode output was recorded with a transient digitizer. The shape of the input pulse was similar to that shown in Fig. 4. Figure 9 displays pump pulse temporal shapes that have been transmitted through the $\mathrm{Yb}$ :S-FAP rod at four different input fluences.

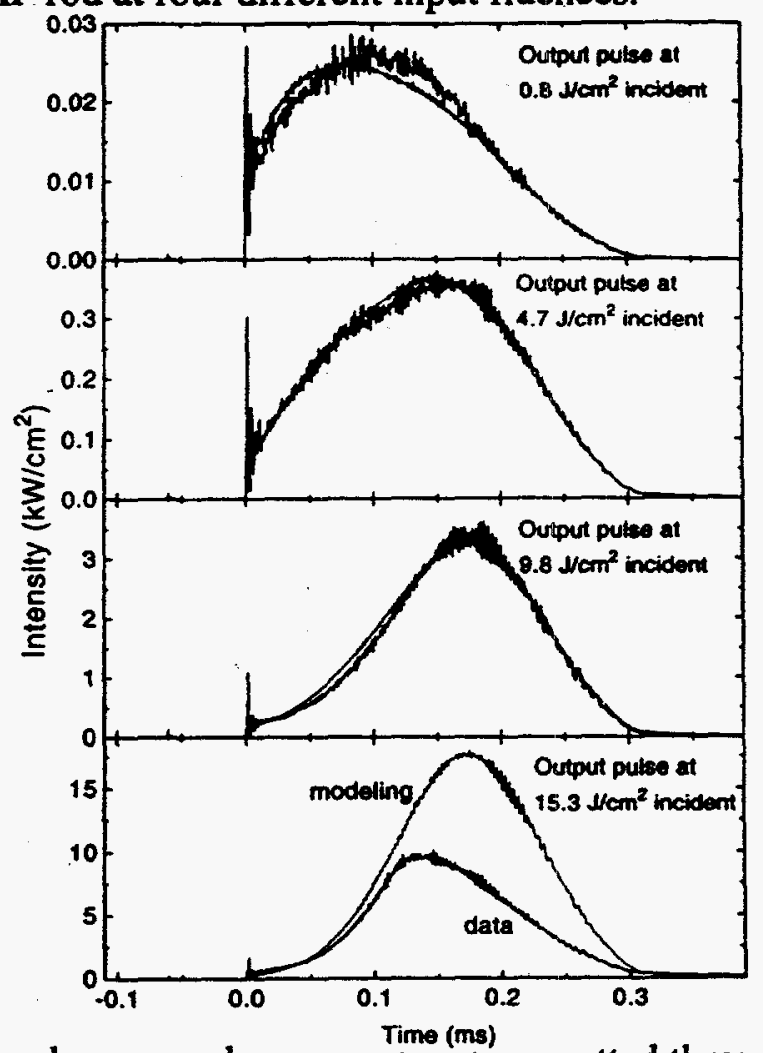

Figure 9. Predicted and measured pump puises transmitted through a $44 \mathrm{~mm}$ Yb:S-FAP rod at different input fluences as noted. Numerical modeling and data are in good agreement except for the highest fluence case where parasitic modes of the rod reduce the transmission of the experimental pump pulse. 
Utilizing the mathematical formalism and assumptions in the previous section and the experimental input pulse shape in Fig. 4, we predicted the output pulse shaping in absolute units as shown in

Fig. 9. The predicted transmitted temporal pump pulses for $0.8,4.7$, and $9.8 \mathrm{~J} / \mathrm{cm}^{2}$ input fluences agree with experiment. However, the $15.3 \mathrm{~J} / \mathrm{cm}^{2}$ calculation deviates from experiment, which is again consistent with the presence of soft parasitic modes at this relatively high pump fluence. The shape of the deviation for the $15.3 \mathrm{~J} / \mathrm{cm}^{2}$ case can be qualitatively understood by recognizing that the excited state population is essentially clamping above $10 \mathrm{~J} / \mathrm{cm}^{2}$. This gives a relatively higher ground state population and a reduced transmission of the pump beam for the trailing end of the pulse from that expected in the absence of parasitic modes. Below $10 \mathrm{~J} / \mathrm{cm}^{2}$ of pump fluence (or below $60 \%$ spatially averaged inversion of the $\mathrm{Yb}^{3+}$ population), the input pump fluence dependence of the transmitted pump pulse distortion, is again consistent with the assumption that parasitics do not play an important role.

Small signal gain

The Yb:S-FAP small signal gain was also measured under the same excitation conditions described above in the fluence-dependent pump transmission section by observing the single pass gain of a weak $-20 \mathrm{~mW} \mathrm{cw}$ Nd:YLF probe laser that was either $\sigma$ or $\pi$ polarized. A Nd:YLF laser was chosen as the probe beam since the polarized emission spectrum of Yb:S-FAP overlaps extremely well with the laser output wavelength as shown in Fig. 10. The favorable overlap of the emission spectra is also well suited to a Nd:YLF/Yb:S-FAP MOPA configuration as is planned later this year. The $\mathrm{cw}$ probe beam was counter-propagated relative to the $\mathrm{Cr}$ :LiSAF pump beam with a 1/e diameter of $200 \mu \mathrm{m}$ and traversed through a spatial filter assembly after exiting the Yb:S-FAP crystal to minimize spontaneous emission contributions to the signal. A photodiode, $1047 \mathrm{~nm}$ bandpass filter, and transient digitizer were then used to measure the small signal gain as a function of time. The temporal peak of the gain was determined for each input fluence as shown in Fig. 11. Using a numerical routine governed by Eqs. 5-7, the small signal gain was also modeled. With an input pulse shape similar to that in Fig. 3, as well as absorption cross sections and $\mathrm{Yb}^{3+}$ concentrations determined from the fluence-dependent pump transmission experiment described above, it was possible to fit the $\pi$ and $\sigma$-polarized small-signal gain data with emission cross sections of $6.0 \pm 1.0$ and $1.45 \pm 0.2 \times 10^{-20} \mathrm{~cm}^{2}$, respectively, as indicated by a solid lines in Fig. 11. This is in reasonable agreement with the literature values of the emission cross section of 7.3 and $1.45 \times 10^{-20} \mathrm{~cm}^{2}$ for $\pi$ and $\sigma$-polarizations, respectively, that were previously reported. ${ }^{3}$ 


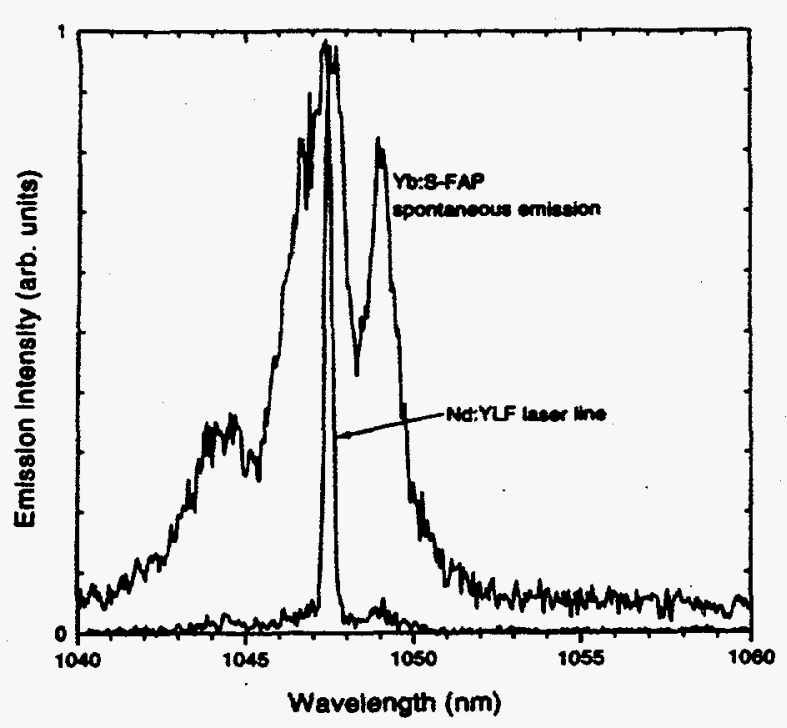

Figure 10. Yb:S-FAP emission spectra overlaid with a diode-pumped $\mathrm{cw}$ Nd:YLF laser emission line. The good overlap at the peak demonstrates that Nd:YLF oscillators can be used as a source to probe and extract the stored energy in Yb:S-FAP amplifiers.

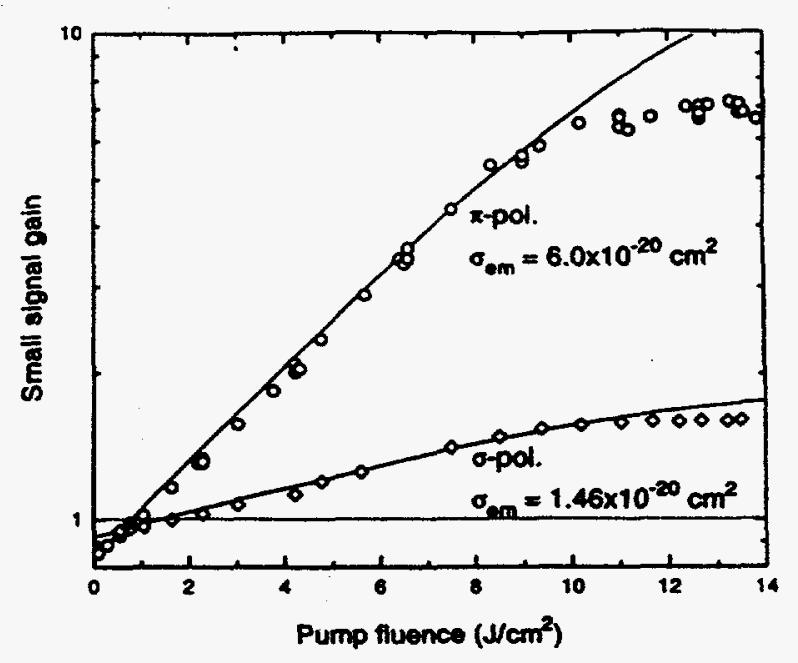

Figure 11. Small signal gain of Yb:S-FAP rod, with either $\sigma$ or $\pi$ polarized at $1047 \mathrm{~nm}$ light as noted, is shown with the same pumping conditions used for Figs. 5, 7, and 9. The emission cross sections obtained from a numerical fit to the data below $10 \mathrm{~J} / \mathrm{cm}^{2}$ are shown as solid lines.

\section{Radiation trapping}

We also investigated the influence of reabsorption on the emission lifetime (i.e. radiation trapping) with a 1047-nm bandpass filter, photodiode, and transient digitizer. The photodiode was not imaged but rather monitored a small solid angle of the total emission from the test sample. In addition to the $6 \times 6 \times 44 \mathrm{~mm}^{3}$ rectangular $\mathrm{Yb}$ :S-FAP crystal used in the above experiments, we also measured the lifetimes of several other $\mathrm{Yb}: \mathrm{S}$-FAP samples ranging in size from a $\sim 0.1 \mathrm{~mm}$ thick crystalline chip to a $50 \mathrm{~mm}$ long S-FAP crystal, with a variety of $\mathrm{Yb}^{3+}$ concentrations.

Figure 12 gives the spontaneous emission as a function of time for two different Yb:S-FAP samples plotted on a $\log _{10}$ scale after excitation with a weak $\left(0.1 \mathrm{~J} / \mathrm{cm}^{2}\right)$ 900-nm pulse from the Cr:LiSAF laser. The data in part (a) were taken on a small chip of material approximately $0.1 \times 1 \times 1 \mathrm{~mm}$ in size with $0.6 \times 10^{19} \mathrm{Yb}^{3}+/ \mathrm{cm}^{3}$ while the data in 


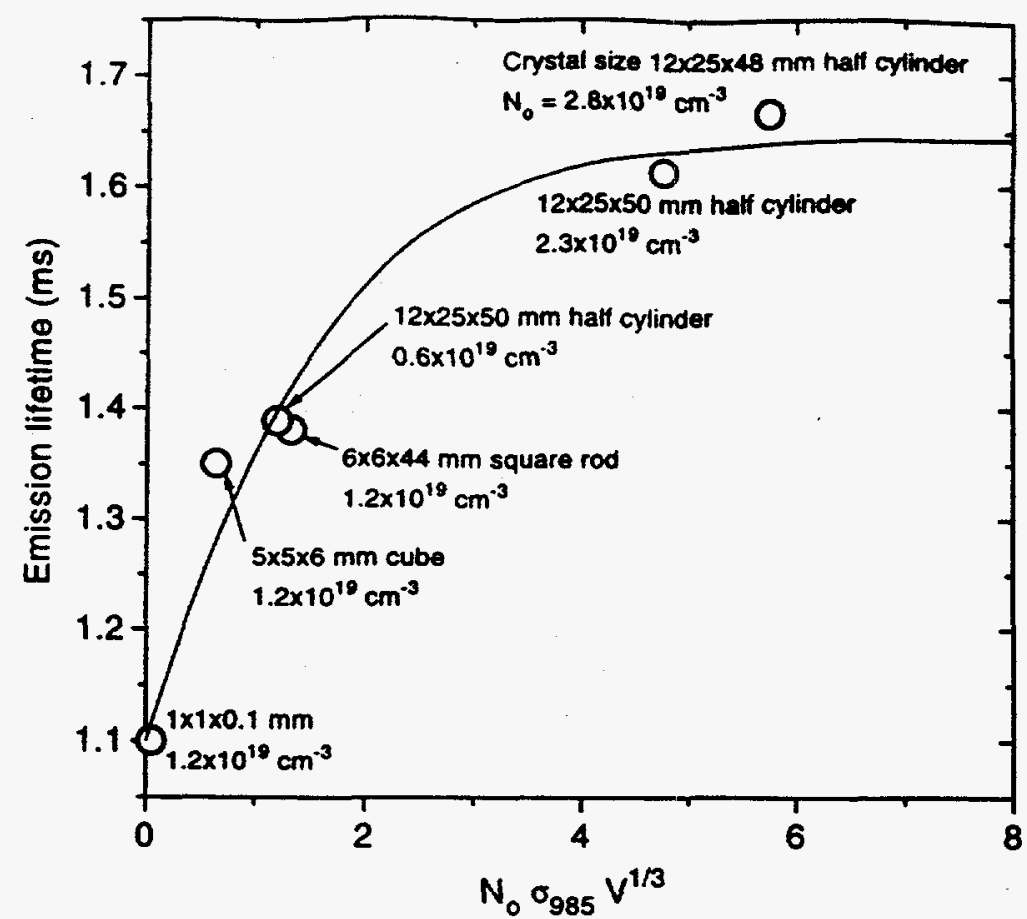

Figure 13. $\mathrm{Yb}: \mathrm{S}-\mathrm{FAP}$ emission lifetime verses the product of the $\mathrm{Yb}^{3+}$ number density $N_{o}$, polarization averaged $985-\mathrm{nm}$ absorption cross section $\sigma_{985}$, and cube root of the crystal volume $V$, for six different crystal shapes and $\mathrm{Yb}^{3+}$ concentrations. The solid line is a fit to the data using a radiation trapping model.

theoretical treatments are available ${ }^{15}$ for better defined geometries, such as polished cubes, that are experimentally planned in the future. In its simplest form, the measured lifetime $\tau_{\text {meas }}$ integrated over the entire medium can be written as 13,14

$\tau_{\text {meas }}=\frac{\tau_{0}}{1-\beta f_{a b s}}$

where $\tau_{0}$ is the intrinsic emission lifetime of $1.10 \mathrm{~ms}$ (Fig. 12a), $\beta$ is an adjustable parameter closely related to the effective branching ratio into the 985 emission line for $\mathrm{Yb}$ :S-FAP, and $f_{\text {abs }}$ is the fraction of emitted light that is reabsorbed. The fraction reabsorbed into the $985 \mathrm{~nm}$ zero-line can be written as:

$f_{a b s}=1-\exp \left[-N_{g r} \sigma_{985} \gamma V^{1 / 3}\right]$

where $\mathrm{N}_{\mathrm{gr}}$ is the number density of ground-state ions, $\sigma_{985}$ is the absorption coefficient at $985 \mathrm{~nm}$, and $\gamma \mathrm{V}^{1 / 3}$ is taken as a characteristic length proportional to the cube root of the crystal volume $\mathrm{V}$.

Equations 9 and 10 were used to fit the data in Fig. 13 with a least squares fitting algorithm where $\beta$ and $\gamma$ were allowed to vary. The other parameters such as the $\mathrm{Yb}^{3+}$ concentration and crystal volume were taken to be those measured and discussed above. The fitted effective branching ratio, $\beta$, was found to be 0.33 which is in close proximity 
to the actual $985 \mathrm{~nm}$ branching ratio of 0.27 obtained from integrating the polarized emission spectra. The larger experimental $\beta$ value is not unreasonable, since the phenomenological inclusion of other transitions will only serve to increase the effective branching ratio. The geometric scaling factor $\gamma=0.86$ also falls into a reasonable range which implies that an average emission ray length in the irregular crystal geometries is $86 \%$ of the cube root of the volume. A more detailed treatment of the radiation trapping phenomenon would require Monte Carlo ray tracing, which was regarded as beyond the scope of our current work.

The effects of reabsorption were further studied under the influence of significant ground-state bleaching. Using the same experimental setup as the fluence-dependent pump transmission experiment, the emission lifetime was recorded from the same $6 \times 6 \times 44 \mathrm{~mm}$ rod used in the experiments described above and plotted as a function of pump fluence in Fig. 14. The effects of ground-state bleaching, which leads to a sloped emission lifetime verses pump fluence, are clearly present at pump fluences far below the known parasitic amplification threshold of $-10 \mathrm{~J} / \mathrm{cm}^{2}$ described above. The solid line through the data is a numerical fit described below. A similar experiment was also performed on a small $\sim 0.1 \mathrm{~mm}$ thick chip of $\mathrm{Yb}: \mathrm{S}$-FAP. There was no observable change in the emission lifetime from this small sample even at the highest pump fluences of

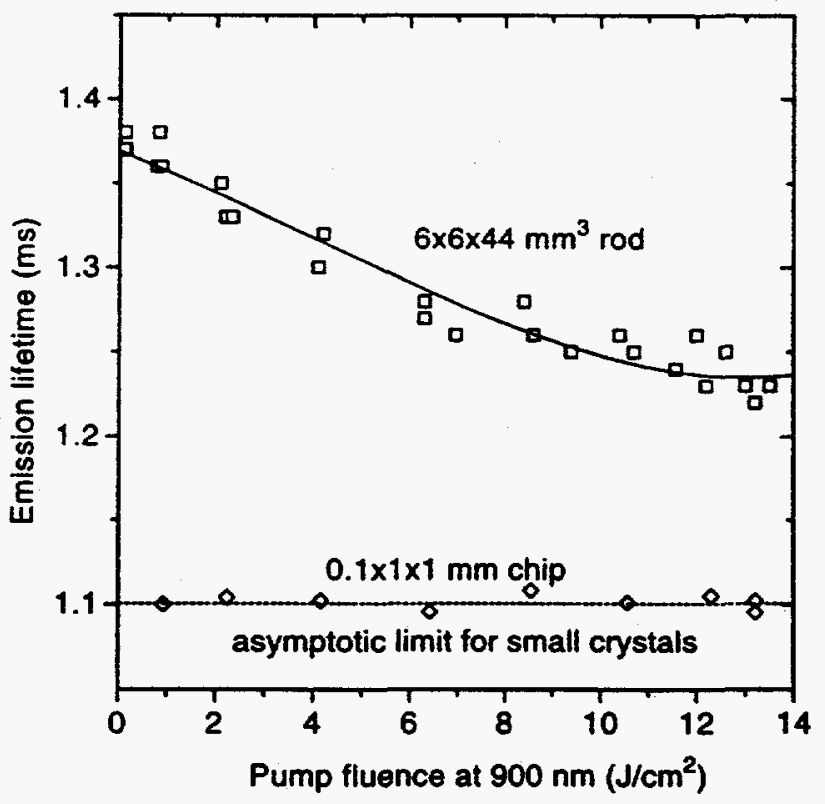

Figure 14. Yb:S-FAP emission lifetime of a $6 \times 6 \times 44$ rod (squares) and a $\sim 0.1 \mathrm{~mm}$ thick chip (diamonds) as a function of pump fluence. The rod experiences a reduction in radiation-trapped lifetime effects due to bleaching at the larger fluences, while the chip shows no signs of radiation trapped effects. 
$14 \mathrm{~J} / \mathrm{cm}^{2}$ which corresponds to $-90 \%$ bleaching of the ground-state. This demonstrates that reabsorption does not play a significant role in determining the lifetime of this chip, and that the measured $1.10 \mathrm{~ms}$ decay is the intrinsic lifetime.

To model the data taken using the $6 \times 6 \times 44 \mathrm{~mm}$ rod in Fig. 14, the pump fluencedependent ground-state number density needs to be included in Eq. 10. The ground-state number density as a function of pump fluence $F$ can be written as:

$$
N_{g r}(F)=N_{g r}^{o}\left(1-\varepsilon f_{i n v}(F)\right)
$$

where $\mathrm{f}_{i n v}$ is the fraction of ions that are inverted as determined experimentally from the bleach-wave experiment described above (Fig. 8), and $\varepsilon$ is a geometric factor that accounts for the pumped verses unpumped crystal regions which is assumed to be the cube root of the ratio of pumped to unpumped volumes. Using the inverted fraction information from Fig. 8 without modification and Egs. 9 and 10, a least squares fit was again performed with $\beta, \gamma$, and $\varepsilon$ as adjustable parameters. This produced the solid line fit shown in Fig. 14 for $\beta=0.30, \gamma=0.81$, and $\varepsilon=0.82$. Table II presents this information along with the parameter values obtained from the experiment involving various sample sizes, and also the estimated values. Very good agreement is obtained between the $\beta$ and $\gamma$ values derived from the two separate experiments which adds credibility to this simplified approach for modeling radiation-trapped lifetimes.

Table II. Parameters derived from fits to radiation trapping experiments (see Egs. 9-11).

\begin{tabular}{|l|l|l|l|l|}
\hline Parameter & $\begin{array}{l}\text { Symbol } \\
-\end{array}$ & $\begin{array}{l}\text { Sample size } \\
\text { concentration } \\
\text { experiment }\end{array}$ & $\begin{array}{l}\text { Pump induced } \\
\text { bleaching } \\
\text { experiment }\end{array}$ & Predicted \\
\hline Branching ratio & $\beta$ & $0.33 \pm 0.02$ & $0.30 \pm 0.1$ & 0.27 \\
\hline $\begin{array}{l}\text { Geometric } \\
\text { factor relating } \\
V^{1 / 3} \text { to } \\
\text { effective length }\end{array}$ & $\gamma$ & $0.86 \pm 0.2$ & $0.81 \pm 0.4$ & $0.5-1.0$ \\
\hline $\begin{array}{l}\text { Geometric } \\
\text { factor relating } \\
\text { peak to average } \\
\text { fraction } \\
\text { inverted }\end{array}$ & $\varepsilon$ & - & $0.82 \pm 0.1$ & $\sim 0.6$ \\
\hline
\end{tabular}

It is relevant to note that the predicted lifetime for a large $\sim 10-\mathrm{cm}$ aperture $\mathrm{kJ}-$ class laser amplifier using slabs of $\mathrm{Yb}: \mathrm{S}-\mathrm{FAP}$ would be expected to be about $1.6 \mathrm{~ms}$ for a typical $\mathrm{Yb}^{3+}$ concentration of $-10^{19} \mathrm{~cm}^{-3}$ based on the model described above. The 
radiation trapping effect is well-known in the context of large Nd:glass amplifier disks where a typical $-0.4 \mathrm{~ms}$ lifetime is observed rather than the intrinsic value of $\sim 0.3 \mathrm{~ms}$. For diode-pumped systems this longer lifetime in Yb:S-FAP significantly lowers the cost of the diode arrays since the required peak pump intensity is lower.

\section{Laser diode array}

The InGaAs laser diode array which we plan to use for the remainder of this year was recently fabricated by $R$. Beach, M. Emanuel and co-workers. This initial array has been tested and qualified to meet or exceed the minimum energy and wavelength specifications for our particular Yb:S-FAP amplifier configuration. The 2D diode array, which consists of 22 micro-channel cooled packages of $1.5 \mathrm{~cm}$ linear diode stripes, ${ }^{16}$ produced $2.8 \mathrm{~J}$ of $900 \mathrm{~nm}$ light per pulse with a $135 \mathrm{~A}$ drive current and a $1 \mathrm{~ms}$ pulse length. The diode output energy of $2.8 \mathrm{~J}$ compares favorably with the $1.2 \mathrm{~J}$ which was available from the Cr:LiSAF oscillator utilized for the experiments described above. The electrical to optical diode efficiency was $\sim 40 \%$. An external lensing-duct ${ }^{16}$ delivery system, which spatially concentrates the diode light by a factor of $\sim 50$, was also fabricated and experimentally observed to have a $75 \%$ transmission efficiency with the diode array described above. The diode array and lensing duct assembly, which fits inside a $4 \times 8 \times 18 \mathrm{~cm}$ compact package, is shown in Fig. 15 and has produced $23 \mathrm{~kW} / \mathrm{cm}^{2}$ of $900 \mathrm{~nm}$ diode pump light in a $1 \mathrm{~ms}$ pulse as shown in Fig. 16. The output pulse yielded a temporally integrated FWHM of $6.3 \mathrm{~nm}$ at a central wavelength of $900.0 \mathrm{~nm}$ when the inlet cooling water temperature was set at $10.6{ }^{\circ} \mathrm{C}$. One requirement is that the laser diode emission band width must be comparable to the relatively narrow $4 \mathrm{~nm}$ FWHM pumping band of Yb:S-FAP during the entire diode pulse length that spans about one emission lifetime of Yb:S-FAP. Figure 17 shows that the diode emission wavelength and bandwidth, taken under the fully loaded conditions described above, overlaps very well with the double-pass transmission spectrum of the 3-cm long Yb:S-FAP amplifier rod with $1.3 \times 10^{19} \mathrm{Yb}^{3+} / \mathrm{cm}^{3}$ that we plan to use for our prototype DPSSL. This is a very positive result and sets aside previous concerns about wavelength shifting of the diode output for the pump conditions of interest over a range that could have been larger than the absorption bandwidth for the pump conditions of interest. Based on this information, we expect that the unbleached Yb:S-FAP rod pumping efficiency will approach $90 \%$. Our future experiments will to be performed on $3 \times 3 \times 30 \mathrm{~mm}$ square rods with dielectric coatings on the small end faces suitable for double-pass pumping and extraction.

Figure 15 on the following page. InGaAs diode array and light pipe concentrator assembly capable of delivering $23 \mathrm{~kW} / \mathrm{cm}^{2}$ at $900 \mathrm{~nm}$. 


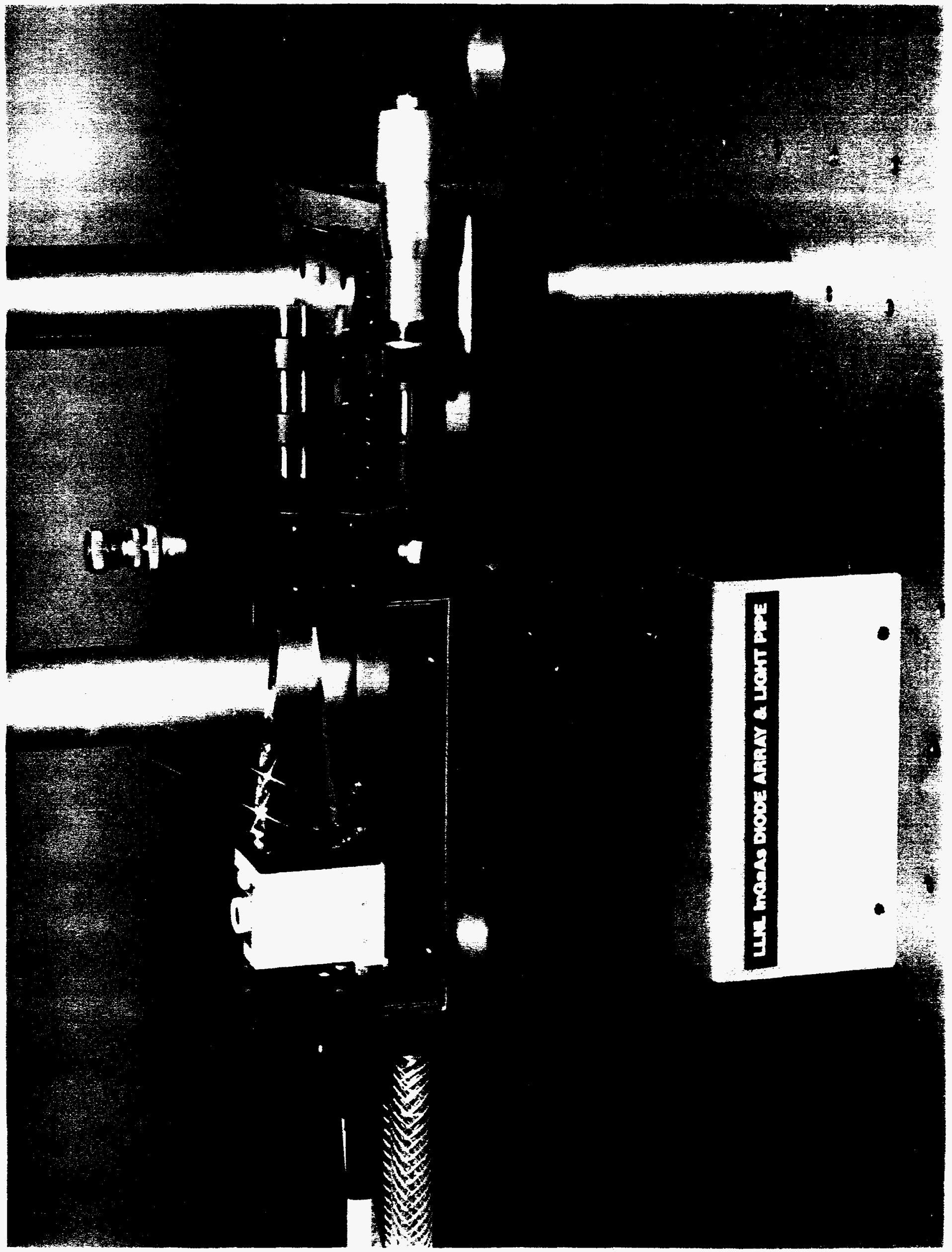




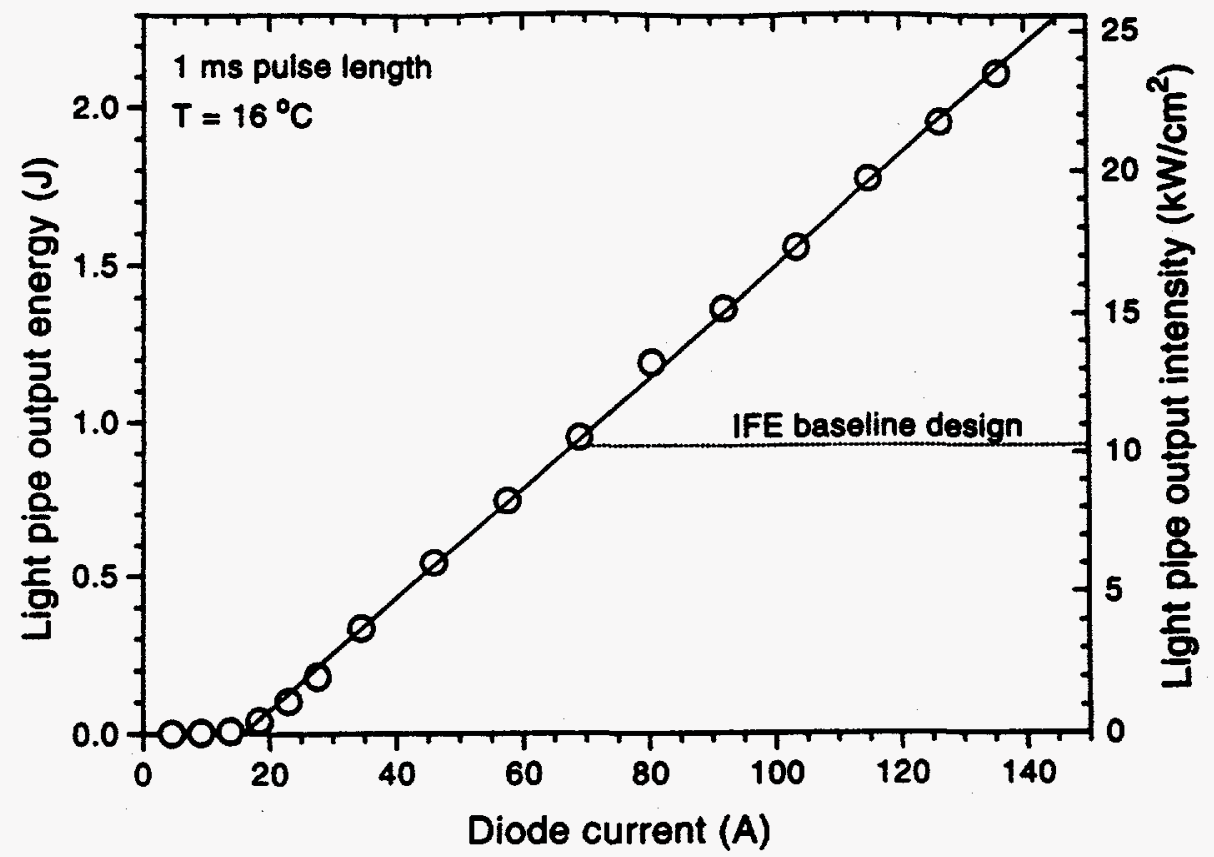

Figure 16. InGaAs diode array energy and intensity verses diode drive current after concentration in a light pipe assembly. The baseline diode intensity in the IFE power plant conceptual design ${ }^{6,7}$ of $10.2 \mathrm{~kW} / \mathrm{cm}^{2}$ is noted for comparison.

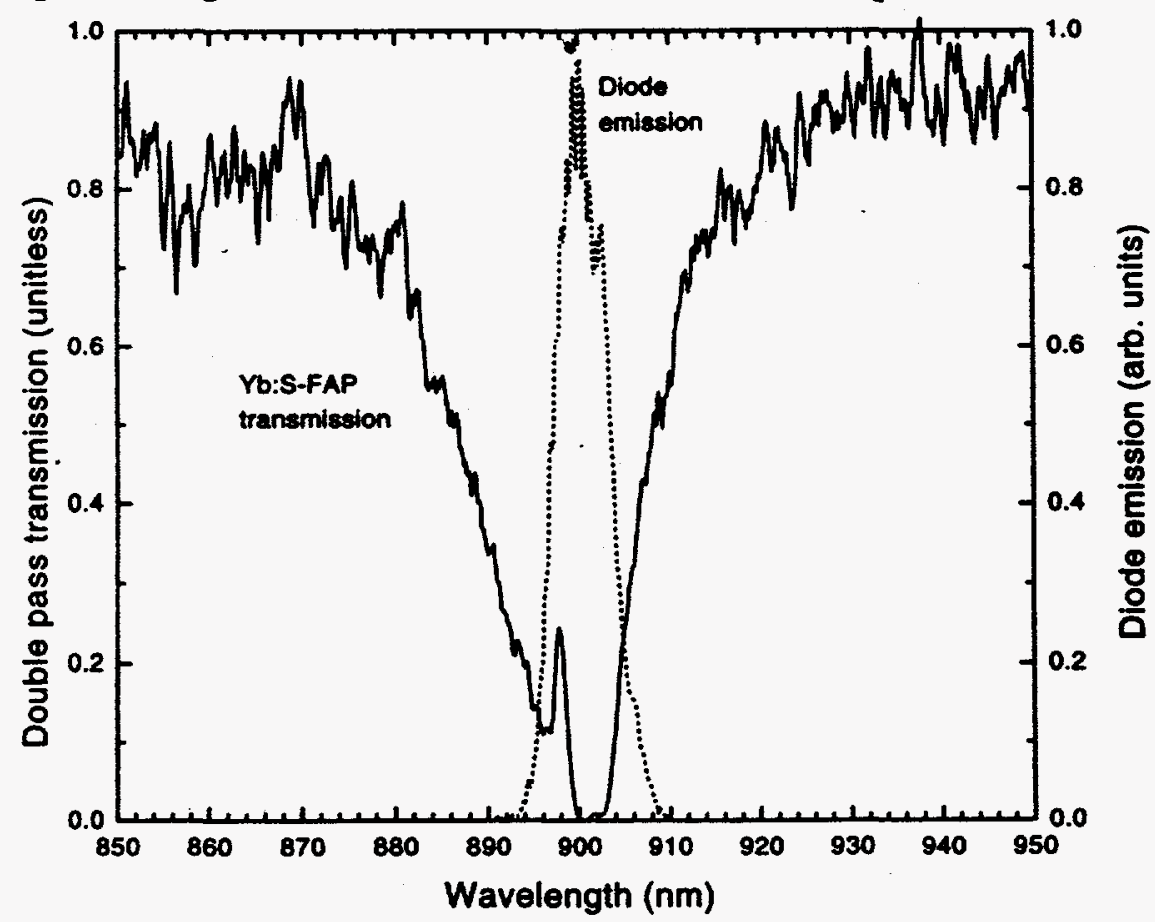

Figure 17. Yb:S-FAP absorption spectra for a double-passed rod with $1.26 \times 10^{19}$ $\mathrm{Yb} / \mathrm{cm}^{3}$ and an experimental InGaAs diode array emission spectra taken at $2.1 \mathrm{~kW}$ peak power output and a $1 \mathrm{~ms}$ pulse length with an input cooling water temperature of $10.6^{\circ} \mathrm{C}$ 


\section{Future Plans}

For the remainder of this year we plan to implement double-pass pumping of $\mathrm{Yb}: S-F A P$ rods with the $2.1 \mathrm{~kW}$ diode array described above. In this configuration small signal gain and saturated extraction efficiency experiments will be performed. The small signal gain will be used to validate the spectroscopically determined emission cross section and the pulsed extraction efficiency experiments will be used to measure the saturation fluence for the extraction process. Our current plans also include the construction of a free-running diode-pumped oscillator from the diode-pumped rod assembly described above. This is expected to produce -1 to $10 \mathrm{~W}$ of average power depending on the maximum repetition rate that thermal management issues will allow. This laser oscillator is expected to operate with $-10 \%$ input-electrical to output-optical efficiency. We also plan to identify a suitable edge cladding for Yb:S-FAP and then, in the upcoming fiscal year devise a means to bond the edge claddings to the Yb:S-FAP gain medium, and test them under an optical absorption induced thermal load. We also plan to implement helium gas-cooled slab technology developed by Albrecht, Sutton and co-workers ${ }^{2}$ on a $\sim 0.2 \times 3 \times 5 \mathrm{~cm}$ Yb:S-FAP slab that will operate as a $-1 \mathrm{~J}$ diode-pumped laser at $\sim 10 \mathrm{~Hz}$ with high $(\sim 10 \%)$ wall plug efficiencies, in order to provide enhanced credibility and visibility for this type of cooling technology. More details of our FY95 plans will be discussed in a forthcoming memo.

\section{Summary}

The initial phase of our experimental campaign to validate Yb:S-FAP as a suitable candidate for laser-driven IFE has been successful. We have demonstrated that high quality crystals can be grown with the necessary loss and laser damage properties. We have experimentally validated our understanding of the saturated pumping dynamics, absorption cross sections, and emission dynamics; we have also been able to quantitatively model the experimental pumping efficiencies. A few important parameters derived from this work are summarized in Table III. A $2.8 \mathrm{~kW}$ peak power laser diode array has been fabricated that meets our wavelength and bandwidth requirements under fully-loaded conditions. All of the elements are in place to proceed this summer with the next phase of experiments to validate the extraction efficiencies of a diode-pumped solidstate optical amplifier based on Yb:S-FAP. 
Table III. Summary of measured Yb:S-FAP experimental parameters.

\begin{tabular}{|l|l|l|}
\hline Parameter & $\begin{array}{l}\text { Measured } \\
\text { values }\end{array}$ & $\begin{array}{l}\text { Reported } \\
\text { values }\end{array}$ \\
\hline $\begin{array}{l}\text { Pump saturation fluence } \\
\left(\mathrm{J} / \mathrm{cm}^{2}\right)\end{array}$ & $2.2 \pm 0.1$ & 2.5 \\
\hline $\begin{array}{l}\text { Intrinsic emission lifetime } \\
(\mathrm{ms})\end{array}$ & $1.10 \pm 0.01$ & 1.26 \\
\hline $\begin{array}{l}\text { Emission cross section } \\
\left(10^{-20} \mathrm{~cm}^{2}\right)\end{array}$ & $6.0 \pm 1.0$ & 7.3 \\
\hline $\begin{array}{l}\text { Radiation trapped lifetime } \\
\text { in highly dense limit (ms) }\end{array}$ & $1.7 \pm 0.05$ & - \\
\hline $\begin{array}{l}\text { Maximum stored energy density } \\
\text { for 6x6x44 mm rod }\left(\mathrm{J} / \mathrm{cm}^{3}\right)\end{array}$ & $1.7 \pm 0.1$ & - \\
\hline $\begin{array}{l}\text { Maximum gain coefficient } \\
\left.\text { for 6x6x44 mm rod (cm }{ }^{-1}\right)\end{array}$ & $0.44 \pm 0.02$ & - \\
\hline
\end{tabular}

\section{Acknowledgments}

We would like to thank L. DeLoach for sharing her technical expertise on the spectroscopic properties of $\mathrm{Yb}$ :S-FAP. We also thank K. Schaffers, J. Tassano, and B. Chai for developing the high quality crystal growth techniques that were essential for the progress described above. We are also indebted to J. Tassano for constructing many of the laboratory facilities and R. Beach, M. Emanuel, and co-workers for successfully fabricating the diode-array described above. We are grateful as well to $R$. Vallene and $P$. Thelin for fabricating and polishing many of the laser crystals used in our experiments, C. Orth for sharing his IFE power plant conceptual design and calculations with us, and H. Powell and B. Krupke for their technical insight and guidance.

\section{References}

1. J. L. Emmett, W. F. Krupke and J. B. Trenholme, Sov. J. Quantum Electron. 13, 1 (1983).

2. S. B. Sutton and G. F. Albrecht, J. Appl. Phys. 69, 1183 (1991 and references therein).

3. L. D. Deloach, S. A. Payne, L. K. Smith, W. L. Kway and W. F. Krupke, J. Opt. Soc. Am. B 11, 269 (1994).

4. L. D. Deloach, S. A. Payne, L. L. Chase, L. K. Smith and W. F. Krupke, IEEE J. of Quantum Electronics 29, 1179 (1994). 
5. L. L. Chase, M. W. Guinan, W. F. Krupke, W. R. Sooy and J. G. Woodworth, A final optics concept for laser driven inertial fusion applications (LLNL internal memorandum, Nov. 25, 1991, 1991).

6. C. D. Orth, S. A. Payne and W. F. Krupke, Inertial Confinement Fusion Quarterly Report 3, 145 (1993).

7. C. D. Orth, S. A. Payne and W. F. Krupke, Nuclear Fusion in-preparation (1994).

8. W. F. Krupke and L. L. Chase, Optical and Quantum Electronics 22, S1 (1990).

9. K. Schaffers, Annealing Yb:S-FAP to reduce scatter loss (LLNL internal memorandum, to be distributed, 1994).

10. DeShazer, Opt. Lett. 13, 363 (1988).

11. A. Yariv and P. Yeh, Optical waves in crystals (Wiley, New York, 1984).

12. A. Erlandson, (personnal communication, 1994).

13. J. B. Birks, Phys. Rev. 94, 1567 (1954).

14. J. A. Caird, A. J. Ramponi and P. R. Staver, J. Opt. Soc. Am. B 8, 1391 (1991).

15. T. Holstein, Phys. Rev. 72, 1212 (1947).

16. R. J. Beach and e. al., "Talk 2148-02, January 24", Laser diode technolgy and applications VI conference, SPIE OE Lase Symposium, Los Angeles, CA (1994). 